\title{
Bicyclische Lithium-t-butylaminofluorsilane, Moleküle mit zwei- und vierfachkoordiniertem Lithium
}

\author{
D. Stalke, U. Klingebiel ${ }^{\star}$ und G.M. Sheldrick \\ Institut für Anorganische Chemie der Universität Göttingen, Tammannstrasse 4, D-3400 Göttingen (B.R.D.)
}

(Eingegangen den 18. September 1987)

\begin{abstract}
Dimeric lithium-t-butylamino-di-t-butylfluorosilane, $\left[\left(\mathrm{CMe}_{3}\right)_{2} \mathrm{SiFLiNCMe}_{3}\right]_{2}$, adds one molecule of THF per dimer when crystallized from THF. A bicyclic compound is obtained with fourfold $\left[\mathrm{F}_{2} \mathrm{Li}(\mathrm{THF}) \mathrm{N}\right]$ and twofold [NLiN] coordinated lithium. The $\mathrm{Si}(1)-\mathrm{N}(1)$ bond length in the six-membered ring has the length expected for a $\mathrm{Si}=\mathrm{N}$ bond and is $5.4 \mathrm{pm}$ shorter than the $\mathrm{Si}(2)-\mathrm{N}(2)$ bond of the four-membered ring. The ${ }^{7} \mathrm{Li}$ and the solid state ${ }^{13} \mathrm{C}$ NMR spectra confirm the asymmetry of the molecule. NMR spectra in solution show an equilibration of the $\mathrm{H}, \mathrm{C}, \mathrm{F}$ and $\mathrm{Si}$ atoms, a feature which is consistent with fluctuation of $\mathrm{Li}(1)-\mathrm{N}(1)$ and $\mathrm{Li}(1)-\mathrm{N}(2)$ bonds. The THF adduct of lithiated t-butylamino-bis(methyltrimethylsilylamino)-fluorosilane exhibits similar NMR effects.
\end{abstract}

\section{Zusammenfassung}

Dimeres Lithium-t-butylamino-di-t-butylfluorsilan, $\left[\left(\mathrm{CMe}_{3}\right)_{2} \mathrm{SiFLiNCMe}_{3}\right]_{2}$, addiert beim Kristallisieren aus THF pro Dimer ein Molekül THF. Es entsteht ein Bicyclus mit Lithium in Vier- $\left[\mathrm{F}_{2} \mathrm{Li}(\mathrm{THF}) \mathrm{N}\right]$ und Zweifachkoordination [NLiN]. Die Si(1)-N(1)-Bindungslänge des sechsgliedrigen Ringes hal die Grössenordnung einer $\mathrm{Si}=\mathrm{N}$-Bindung und ist $5.4 \mathrm{pm}$ kürzer als die $\mathrm{Si}(2)-\mathrm{N}(2)$-Bindung des viergliedrigen Ringes. ${ }^{7} \mathrm{Li}$ - und Festkörper- ${ }^{13} \mathrm{C}-\mathrm{NMR}$-Aufnahmen bestätigen die Asymmetrie des Moleküls. Eine Äquilibrierung der H-, C-, F- und Si-Atome verdeutlichen LösungsNMR-Aufnahmen, ein Befund, der mit fluktuierender Li(1)-N(1)- und Li(1)-N(2)Bindung zu erklären ist. Vergleichbare NMR-Daten liefert das THF-Addukı des lithiierten t-Butylamino-bis(methyl-trimethylsilylamino)-fluorsilan, [( $\left.\mathrm{Me}_{3} \mathrm{SiNMe}\right)_{2}-$ $\mathrm{SiFLiNCMe}]_{2} \cdot$ THF.

Zwei bemerkenswerte Eigenschaften haben Lithiumsalze von Aminen und Silylaminen. Gegenüber elektrophilen Zentren fungieren sie als starke Lewis-Basen und gegenüber Donor-Solventien als Lewis-Säuren [1]. Eine Folge dieser Eigenschaften 
ist häufig die Oligomerisation der Lithiumsalze im kristallinen Zustand. Der Grad der Oligomerisation wird massgeblich von der Grösse der am Stickstoff gebundenen Organyl- bzw. Silylgruppen sowie vom verwendeten Lösungsmittel beeinflusst. Nucleophile Lösungsmittel, z.B. Ether führen aufgrund ihrer Bindung an das Lithiumatom in der Regel zur Erniedrigung des Oligomerisationsgrades [1,2].

Überraschungen bieten ebenfalls die Koordinationszahlen der Stickstoff- und Lithiumatome. Der Stickstoff erreicht in einigen Salzen eine Fünferkoordination [3]. Da die Valenzschale des $\mathrm{N}$-Atoms jedoch nur vier Elektronenpaare aufnehmen kann, muss hier eine Elektronendefizit-Bindung existent sein. Das Li-Atom erreicht in einigen Salzen seine niedrigste, die Zweierkoordination [1,4].

Erste Lithiumsalze von lithiierten Aminofluorsilanen sind seit ca. 10 Jahren bekannt [4] und dienten seit dieser Zeit als vorzüglicher Synthesebaustein zahlreicher neuer Moleküle [5]. Im Gegensatz zu lithiierten Silylaminen, die im kristallinen Zustand aufgrund intermolekulare Wechselwirkung oligomerisieren, wird in diesen Salzen dem Li-Atom zusätzlich intramolekular die Base Fluor angeboten. Dies hat beträchtliche Auswirkungen auf die Kristallstrukturen der Verbindungen [4]. Werden die Salze aus unpolaren Lösungsmitteln synthetisiert, übernimmt das Fluoratom häufig die Funktion eines Donormoleküls, es entstehen tricyclische, dimere Moleküle [4].<smiles>CN1[Al]2[I-][Si](C)(C)N2[Si]1(C)C</smiles><smiles>[2H][I]1([2H])[I-][Si](C)(C)N1C</smiles>

In Donorsolventien (D), z.B. THF, wird der Säure Lithium eine weitere Base zur Koordination angeboten. Dies führt auch bei lithiierten Aminofluorsilanen häufig zur Abnahme des Oligomerisationsgrades, zur Bildung von Monomeren und der Erhöhung der Koordinationszahl des Lithiumatoms [4]. Die mit der Abnahme des Oligomerisationsgrades und der Anlagerung der Donormoleküle verbundene $\mathrm{Li}-\mathrm{N}$ Bindungsspaltung wird elektronisch und sterisch beeinflusst. Hat der Stickstoff eine geringe Basizität, z.B. im Fall gebundener Aromaten, orientiert sich das Li-Atom ausschliesslich zur stärkeren Base Fluor. Es entstehen LiF-Addukte von Iminosilanen [5].<smiles>[2H][Te]([2H])([2H])[Si](C)(C)=NC</smiles>

Im Rahmen dieser Arbeit diskutieren wir anhand von Struktur- und NMR-Daten den interessanten Fall der Addition eines Donormoleküls pro Dimer.

\section{Ergebnisse und Diskussion}

Die Aminofluorsilane 1 [6] und 2 [7] reagieren in n-Hexan mit $\mathrm{n}-\mathrm{C}_{4} \mathrm{H}_{9} \mathrm{Li}$ unter Bildung der Lithiumsalze 3 [2,6] und 4: 


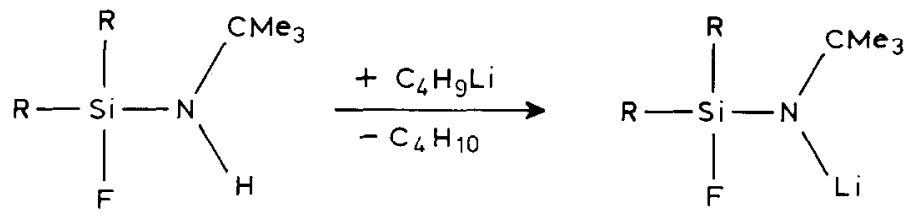
(1 [6], 2[7])
$(3[2,6], 4)$

3 is mässig in n-Hexan löslich und konnte spektroskopisch und strukturanalytisch charakterisiert werden [2]. 3 kristallisiert als tricyclisches Dimer.<smiles>[R][Si]1([R])[I-][Si]2(N1C)N(C(C)(C)C)[Si]2([R])[R]</smiles>

(3)

Werden 3 und 4 in THF umkristallisiert, addieren sie jeweils pro Dimer ein Molekül THF unter Bildung von 5 und 6. Von 5 konnten wieder Einkristalle gezogen und eine Strukturanalyse durchgeführt werden, so dass hier erstmals die strukturverändernde Wirkung einer Basenanlagerung an ein Lithiumaminofluorsilan studiert werden kann.

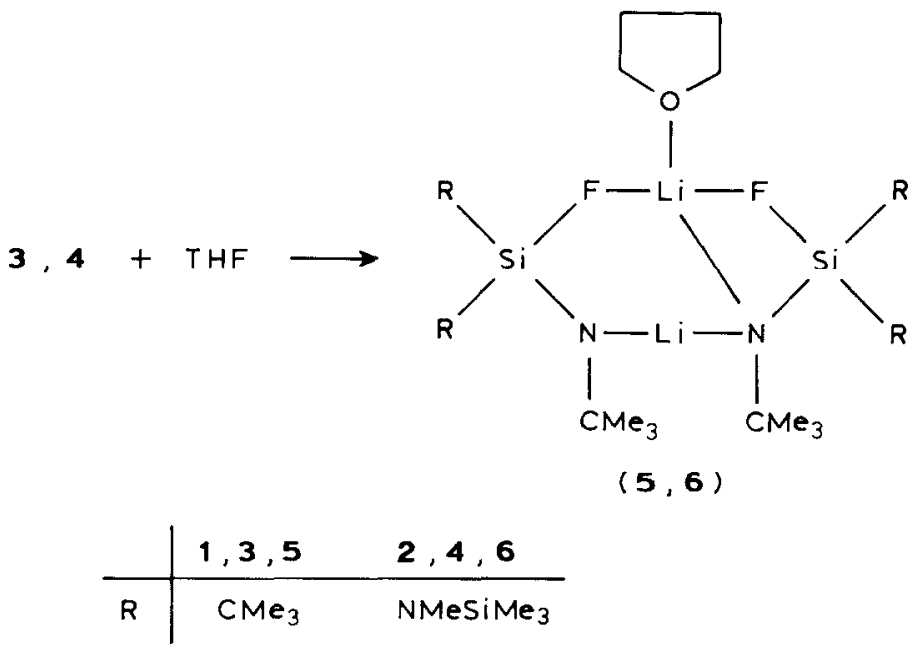

Die Struktur von 5 zeigt einen Bicyclus (Fig. 1). Ein LiNLiNSiF-Sechsring bildet mit einem LiNSiF-Vierring einen Winkel von $105.4^{\circ}$.

Im Kristall liegt ein vierfach koordiniertes Lithiumatom neben cinem zwcifach koordinierten vor. $\mathrm{Li}(1)$ ist mit beiden Fluoratomen des Dimeren, einem Stickstoffatom und einem THF-Molekül verbunden. $\mathrm{Li}(2)$ ist nur mit beiden Stickstoffatomen 


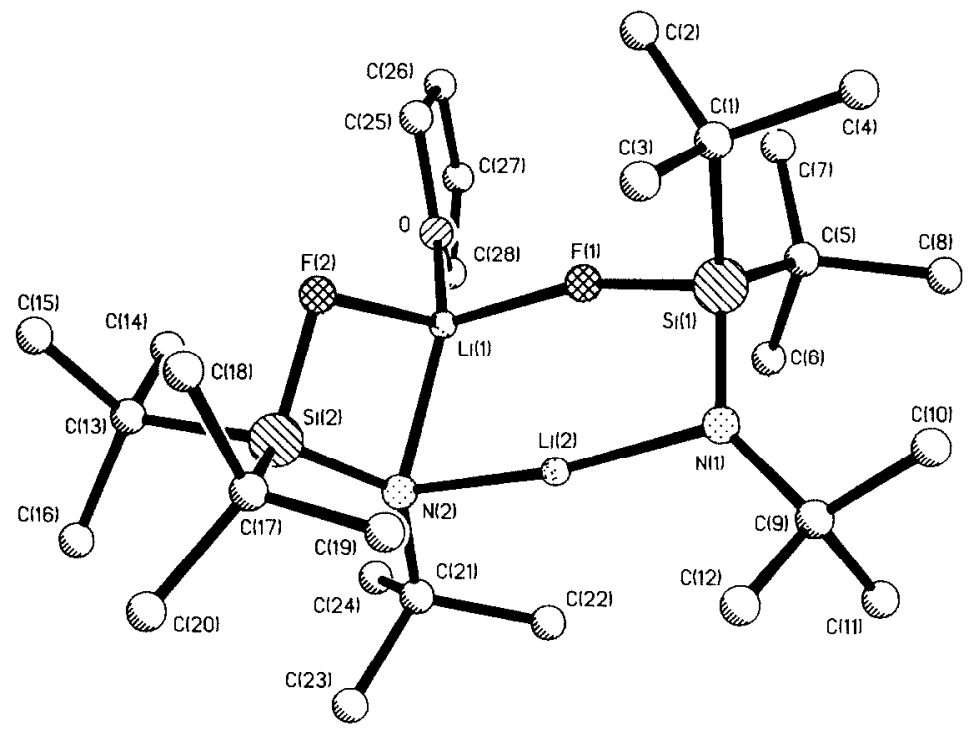

Fig. 1. Die Struktur des Bicyclus 5 im Festkörper.

des Dimeren koordiniert. Die $\operatorname{Si}(1)-\mathrm{N}(1)$-Bindung ist mit $162.7 \mathrm{pm}$ um $5.4 \mathrm{pm}$ kürzer als die $\mathrm{Si}(2)-\mathrm{N}(2)$-Bindung (Tab. 2) und im Bereich der $\mathrm{Si}=\mathrm{N}$-Bindung von Iminosilanen. Die mittlere Si-F-Bindung beträgt $166.3 \mathrm{pm}$.

Die Winkel $\mathrm{N}(1)-\mathrm{Li}(2)-\mathrm{N}(2)$ beträgt $166.3^{\circ}$ (Tab. 3). Somit liegt ein fast linear koordinicrtes neben einem tetraedrisch koordinierten Lithiumatom vor. Der $\mathrm{Li}(1)-\mathrm{F}(2)$-Abstand ist mit 208.7 pm deutlich länger als der $\mathrm{Li}(1)-\mathrm{F}(1)$-Abstand mit $185.1 \mathrm{pm}$. Auch die Li(2)-N-Abstände weisen einen Unterschied von $7.2 \mathrm{pm}$ auf. Da $N(2)$ schon durch $\operatorname{Li}(1)$ koordiniert ist, bildet $\mathrm{N}(1)$ für $\mathrm{Li}(2)$ die stärkere Lewis-Base und bindet $\operatorname{Li}(2)$ stärker als $N(2)$.

Die t-Butylgruppen bilden für das $\mathrm{Li}(2)$ eine Taschc. Es kommt zu einem in Lithium-Stickstoff-Verbindungen ungewöhnlich kurzem Li-H-Kontakt. Der Abstand $\mathrm{Li}(2) \mathrm{zu}$ einem Methylwasserstoffatom am C(22) beträgt $212 \mathrm{pm}$ und liegt damit im Bereich der gleichen Distanz in $\mathrm{Me}_{4} \mathrm{BLi}$ [8].

Der Si-N-C-Winkel der kürzeren Si-N-Bindung ist in Richtung Iminosilanwinkel aufgeweitet (139.1 ${ }^{\circ}$ gegenüber $\left.130.8^{\circ}\right)$.

Die Atomkoordinaten, weitere Bindungslängen und -winkel können den Tabellen 1-3 entnommen werden. Wichtige kristallographische Daten sind in Tab. 4 zusammengestellt.

Die Reflexintensitäten wurden auf einem Stoe-Siemens-AED-Diffraktometer mit graphitmonochromatisierter Mo- $K_{\alpha}$-Strahlung ( $\lambda 71.069 \mathrm{pm}$ ) gesammelt. Die Struktur wurde mit Hilfe "Direkter Methoden" gelöst und nach dem Kleinste-QuadrateVerfahren mit allen Nicht-Wasserstoffatomen verfeinert. Wasserstoffatome wurden geometrisch ideal positioniert und nach einem Reitermodell mit festen isotropen Thermalparametern verfeinert. Das Gewichtsschema betrug $w^{-1}=\sigma^{2}(F)+g F^{2}$; der $g$-Wert ist in Tabelle 4 angegeben.

Weitere Einzelheiten können beim Fachinformationszentrum Energie, Physik, Mathematik, D-7514 Fggenstein-Leopoldshafen 2, unter Angabe der Hinterle- 
Tabelle 1

Atomkoordinaten $\left(\times 10^{4}\right)$ und äquivalente isotrope Thermalparameter $\left(\mathrm{pm}^{2} \times 10^{-1}\right)$ von 5

\begin{tabular}{|c|c|c|c|c|}
\hline Atom & $x$ & $y$ & $z$ & $U_{\text {eq }}{ }^{a}$ \\
\hline Si(1) & $2306(3)$ & $1997(3)$ & $1071(2)$ & $28(1)$ \\
\hline $\operatorname{Si}(2)$ & $3367(3)$ & $2571(3)$ & $-1203(2)$ & $26(1)$ \\
\hline$F(1)$ & $2662(5)$ & $1444(5)$ & $407(3)$ & $32(2)$ \\
\hline$F(2)$ & $3973(4)$ & $1881(6)$ & $-609(3)$ & $32(2)$ \\
\hline$N(1)$ & $1791(7)$ & $3100(8)$ & $773(4)$ & $28(3)$ \\
\hline $\mathrm{N}(2)$ & $2251(7)$ & $2323(7)$ & $-1009(4)$ & $18(2)$ \\
\hline $\operatorname{Li}(1)$ & $2752(16)$ & $1000(16)$ & $-433(9)$ & $28(7)$ \\
\hline $\operatorname{Li}(2)$ & $2033(15)$ & $2906(16)$ & $-126(9)$ & $28(7)$ \\
\hline $\mathrm{O}$ & $2839(6)$ & $-525(7)$ & $-454(4)$ & $35(3)$ \\
\hline $\mathrm{C}(1)$ & $3500(9)$ & $2113(11)$ & $1582(6)$ & $37(5)$ \\
\hline$C(2)$ & $4119(11)$ & $1114(14)$ & $1531(7)$ & $71(7)$ \\
\hline$c(3)$ & $4003(10)$ & $3118(13)$ & $1359(7)$ & $66(6)$ \\
\hline$C(4)$ & $3369(11)$ & $2230(12)$ & $2311(6)$ & $59(6)$ \\
\hline$C(5)$ & $1510(10)$ & $853(11)$ & $1315(5)$ & $36(5)$ \\
\hline$C(6)$ & $734(10)$ & $647(13)$ & $751(7)$ & $63(6)$ \\
\hline$C(7)$ & $2030(12)$ & $-240(12)$ & $1471(8)$ & $66(6)$ \\
\hline$C(8)$ & $953(10)$ & $1146(13)$ & $1903(7)$ & $59(6)$ \\
\hline$C(9)$ & $1263(11)$ & $4049(11)$ & $990(6)$ & $43(5)$ \\
\hline $\mathrm{C}(10)$ & $1499(10)$ & $4272(12)$ & $1705(6)$ & $47(6)$ \\
\hline$C(11)$ & $157(11)$ & $3829(13)$ & $879(7)$ & $61(5)$ \\
\hline $\mathrm{C}(12)$ & $1507(14)$ & $5048(13)$ & $630(8)$ & $90(8)$ \\
\hline C(13) & $3859(10)$ & $1851(11)$ & $-1925(6)$ & $40(5)$ \\
\hline$C(14)$ & $3666(9)$ & $594(12)$ & $-1870(7)$ & $44(5)$ \\
\hline$C(15)$ & $4946(12)$ & $2004(15)$ & $-1893(7)$ & $74(7)$ \\
\hline C(16) & $3338(11)$ & $2241(14)$ & $-2589(6)$ & $62(7)$ \\
\hline$C(17)$ & $3814(9)$ & $3997(10)$ & $-1072(6)$ & $30(4)$ \\
\hline$C(18)$ & $4907(10)$ & $4035(13)$ & $-806(7)$ & $54(5)$ \\
\hline C(19) & $3261(12)$ & $4556(11)$ & $-559(6)$ & $56(6)$ \\
\hline$C(20)$ & $3723(12)$ & $4736(12)$ & $-1670(7)$ & $59(6)$ \\
\hline$C(21)$ & $1330(9)$ & $2420(11)$ & $-1388(6)$ & $33(5)$ \\
\hline$C(22)$ & $561(10)$ & $2527(13)$ & $-918(6)$ & $51(6)$ \\
\hline$C(23)$ & $1263(9)$ & $3419(10)$ & $-1828(7)$ & $38(5)$ \\
\hline$C(24)$ & $1076(10)$ & $1395(11)$ & $-1790(6)$ & $43(5)$ \\
\hline$C(25)$ & $3573(13)$ & $-1141(13)$ & $-137(9)$ & $84(7)$ \\
\hline$C(26)$ & $3208(24)$ & $-2266(18)$ & $-83(23)$ & $294(18)$ \\
\hline $\mathrm{C}(27)$ & $2391(21)$ & $-2401(18)$ & $-535(17)$ & $167(14)$ \\
\hline $\mathrm{C}(28)$ & $2120(15)$ & $-1301(17)$ & $-696(11)$ & $105(9)$ \\
\hline
\end{tabular}

a Aquivalente isotrope $U$ berechnet als ein Drittel ein Spur des orthogonalen $U_{i j}$ Tensors.

gungsnummer CSD 52667 und des vollständigen Zeitschriftenzitats angefordert werden.

Von der Verbindung 5 wurde ein ${ }^{13} \mathrm{C}$-Festkörper-NMR-Spektrum mit 'MagicAngle-Spinning' angefertigt Die Probe wurde unter Argonatmosphäre präpariert. Dieses Spektrum (Fig. 2) bestätigt den Befund der Kristallstruktur. Geminale $\mathrm{SiCC}_{3}$-Kerne liefern zwei Signale, die nicht durch eine Kopplung erklärt werden können. Auch die $\mathrm{NCC}_{3}$-Kerne sind nicht äquivalent. Im Spektrum sind die Signale geminaler (d.h. $\mathrm{C}(1), \mathrm{C}(17)$ und $\mathrm{C}(5), \mathrm{C}(13)$ ) tertiärer C-Atome am Si zu unterscheiden. Auch die NC-Kerne (Tab. 5) liefern zwei Signale, wobei nicht mit letzter Sicherheit auszuschliessen ist, dass es sich hier um quadrupolare Restauf- 


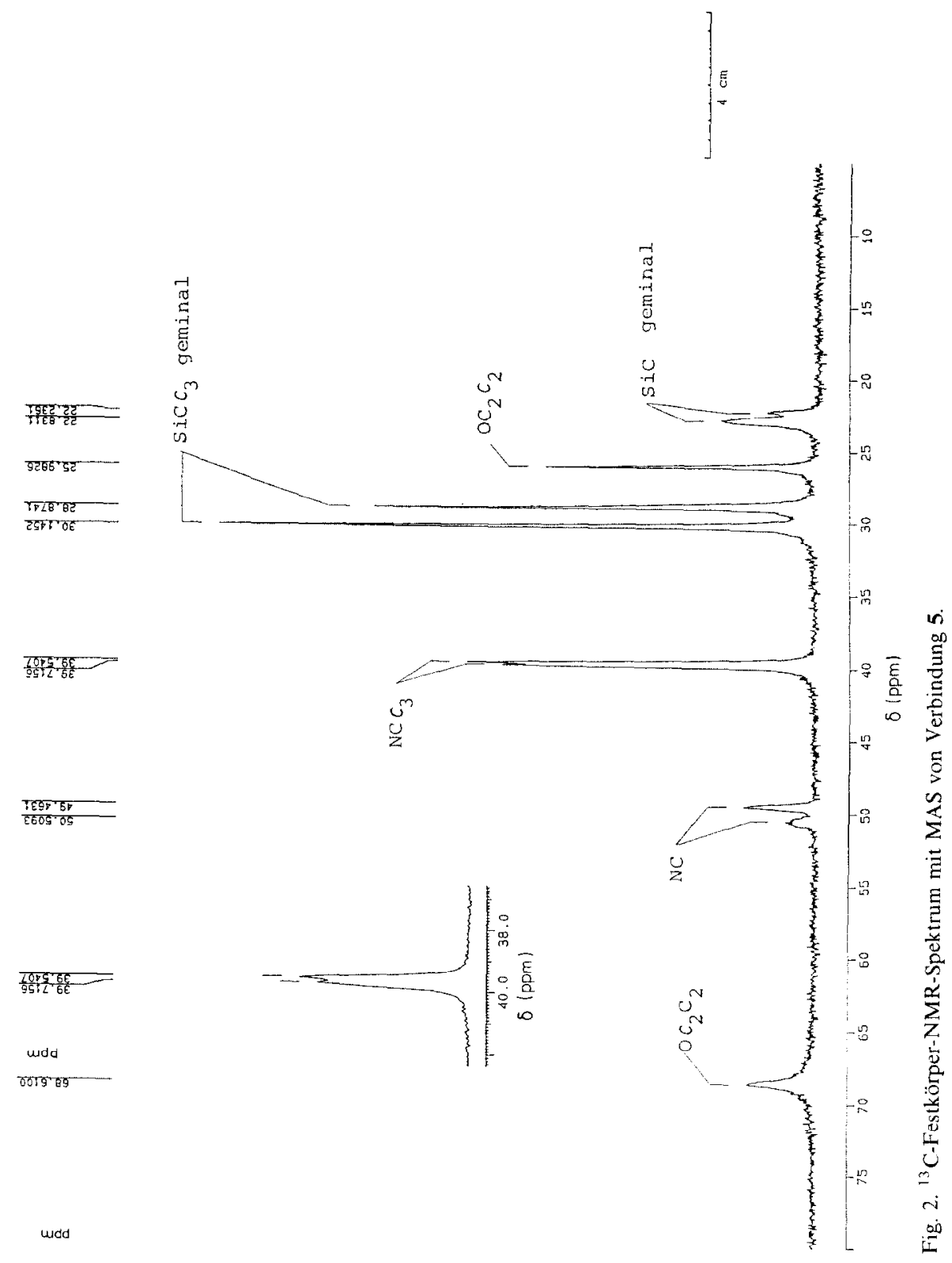


Tabelle 2

Bindungsabstănde (pm) von 5

\begin{tabular}{llll}
\hline $\mathrm{Si}(1)-\mathrm{F}(1)$ & $165.9(7)$ & $\mathrm{Si}(1)-\mathrm{N}(1)$ & $162.7(10)$ \\
$\mathrm{Si}(1)-\mathrm{C}(1)$ & $190.8(13)$ & $\mathrm{Si}(1)-\mathrm{C}(5)$ & $189.1(14)$ \\
$\mathrm{Si}(2)-\mathrm{F}(2)$ & $166.7(7)$ & $\mathrm{Si}(2)-\mathrm{N}(2)$ & $168.1(10)$ \\
$\mathrm{Si}(2)-\mathrm{C}(13)$ & $192.7(14)$ & $\mathrm{Si}(2)-\mathrm{C}(17)$ & $186.7(13)$ \\
$\mathrm{F}(1)-\mathrm{Li}(1)$ & $185.1(21)$ & $\mathrm{F}(2)-\mathrm{Li}(1)$ & $208.7(23)$ \\
$\mathrm{N}(1)-\mathrm{Li}(2)$ & $195.2(22)$ & $\mathrm{N}(1)-\mathrm{C}(9)$ & $147.1(17)$ \\
$\mathrm{N}(2)-\mathrm{Li}(1)$ & $210.0(21)$ & $\mathrm{N}(2)-\mathrm{Li}(2)$ & $202.4(21)$ \\
$\mathrm{N}(2)-\mathrm{C}(21)$ & $145.8(15)$ & $\mathrm{Li}(1)-\mathrm{O}$ & $187.2(21)$ \\
$\mathrm{O}-\mathrm{C}(25)$ & $139.3(19)$ & $\mathrm{O}-\mathrm{C}(28)$ & $144.5(22)$ \\
$\mathrm{C}(1)-\mathrm{C}(2)$ & $151.0(21)$ & $\mathrm{C}(1)-\mathrm{C}(3)$ & $151.4(21)$ \\
$\mathrm{C}(1)-\mathrm{C}(4)$ & $155.3(18)$ & $\mathrm{C}(5)-\mathrm{C}(6)$ & $154.8(18)$ \\
$\mathrm{C}(5)-\mathrm{C}(7)$ & $154.5(20)$ & $\mathrm{C}(5)-\mathrm{C}(8)$ & $155.6(19)$ \\
$\mathrm{C}(9)-\mathrm{C}(10)$ & $152.2(18)$ & $\mathrm{C}(9)-\mathrm{C}(11)$ & $157.2(22)$ \\
$\mathrm{C}(9)-\mathrm{C}(12)$ & $149.3(21)$ & $\mathrm{C}(13)-\mathrm{C}(14)$ & $156.8(20)$ \\
$\mathrm{C}(13)-\mathrm{C}(15)$ & $153.2(21)$ & $\mathrm{C}(13)-\mathrm{C}(16)$ & $158.0(18)$ \\
$\mathrm{C}(17)-\mathrm{C}(18)$ & $158.4(19)$ & $\mathrm{C}(17)-\mathrm{C}(19)$ & $153.9(20)$ \\
$\mathrm{C}(17)-\mathrm{C}(20)$ & $153.6(19)$ & $\mathrm{C}(21)-\mathrm{C}(22)$ & $152.9(19)$ \\
$\mathrm{C}(21)-\mathrm{C}(23)$ & $152.7(18)$ & $\mathrm{C}(21)-\mathrm{C}(24)$ & $153.4(19)$ \\
$\mathrm{C}(25)-\mathrm{C}(26)$ & $147.7(29)$ & $\mathrm{C}(26)-\mathrm{C}(27)$ & $142.4(49)$ \\
$\mathrm{C}(27)-\mathrm{C}(28)$ & $143.2(31)$ & & \\
\hline
\end{tabular}

spaltung handeln kann. Dieser Befund konnte mit einem ${ }^{13} \mathrm{C}$-NMR aus Lösung auch bei $-72^{\circ} \mathrm{C}$ nicht reproduziert werden. Auch bei tiefer Temperatur sind die $\mathrm{SiCC}_{3}$ - und $\mathrm{NCC}_{3}$-Gruppen magnetisch äquivalent.

Das ${ }^{7} \mathrm{Li}-\mathrm{NMR}$-Spektrum aus Lösung liefert bei $+32^{\circ} \mathrm{C}$ ein Singulett bei 0.91 ppm. Wird die Probe gekuhlt, so beginnt sich bei $-40^{\circ} \mathrm{C}$ (Fig. 3a) ein zweites Signal abzuzeichnen. Bei $-72^{\circ} \mathrm{C}$ kann man ein Singulett bei $1.33 \mathrm{ppm}$ und ein pseudo-Triplett, das durch ein überlagertes Dublett vom Dublett entsteht, bei -0.18 ppm unterscheiden (Fig. 3b). Die ${ }^{19}$ F-BB-entkoppelte Aufnahme (Fig. 3c)

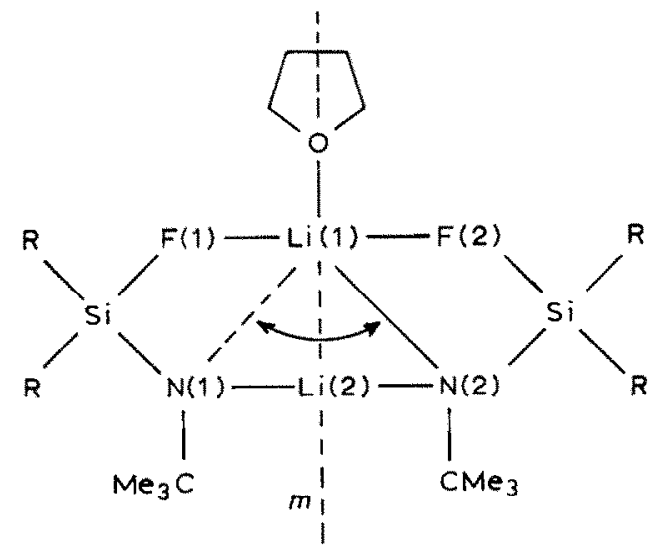

\begin{tabular}{c|cc} 
& 3 & 6 \\
\hline$R$ & CMe $_{3}$ & NMesiMe $_{3}$
\end{tabular}


Tabelle 3

Bindungswinkel $\left({ }^{\circ}\right)$ von 5

\begin{tabular}{|c|c|c|c|}
\hline $\mathrm{N}(1)-\mathrm{Si}(1)-\mathrm{F}(1)$ & $100.2(4)$ & $C(1)-\operatorname{Si}(1)-F(1)$ & $100.4(5)$ \\
\hline$C(1)-\operatorname{Si}(1)-N(1)$ & $119.3(6)$ & $C(5)-\operatorname{Si}(1)-F(1)$ & $98.7(5)$ \\
\hline$C(5)-S i(1)-N(1)$ & $117.7(6)$ & $C(5)-S i(1)-C(1)$ & $114.5(6)$ \\
\hline$N(2)-\operatorname{Si}(2)-F(2)$ & $98.8(4)$ & $C(13)-\operatorname{Si}(2)-F(2)$ & $98.9(5)$ \\
\hline$C(13)-\operatorname{Si}(2)-N(2)$ & $121.0(5)$ & $C(17)-\operatorname{Si}(2)-F(2)$ & $102.9(5)$ \\
\hline$C(17)-\operatorname{Si}(2)-N(2)$ & $116.2(5)$ & $C(17)-\operatorname{Si}(2)-C(13)$ & $113.8(6)$ \\
\hline $\operatorname{Li}(1)-F(1)-\operatorname{Si}(1)$ & $164.2(8)$ & $\mathrm{Li}(1)-\mathrm{F}(2)-\mathrm{Si}(2)$ & $91.2(6)$ \\
\hline $\operatorname{Li}(2)-N(1)-\operatorname{Sil}(1)$ & $99.0(8)$ & $C(9)-N(1)-S i(1)$ & $139.1(8)$ \\
\hline $\mathrm{C}(9)-\mathrm{N}(1)-\mathrm{Li}(2)$ & $121.9(9)$ & $\mathrm{Li}(1)-\mathrm{N}(2)-\mathrm{Si}(2)$ & $90.4(7)$ \\
\hline $\operatorname{Li}(2)-N(2)-\operatorname{Si}(2)$ & $112.2(7)$ & $\operatorname{Li}(2)-N(2)-\operatorname{Li}(1)$ & $79.8(8)$ \\
\hline$C(21)-N(2)-S i(2)$ & $130.8(8)$ & $C(21)-N(2)-\operatorname{Li}(1)$ & $127.4(9)$ \\
\hline $\mathrm{C}(21)-\mathrm{N}(2)-\mathrm{Li}(2)$ & $105.4(9)$ & $F(2)-\operatorname{Li}(1)-F(1)$ & $98.2(9)$ \\
\hline$N(2)-\operatorname{Li}(1)-F(1)$ & $105.5(10)$ & $N(2)-\operatorname{Li}(1)-F(2)$ & $74.8(7)$ \\
\hline $\mathrm{O}-\mathrm{Li}(1)-\mathrm{F}(1)$ & $109.0(10)$ & $O-\operatorname{Li}(1)-F(2)$ & $117.1(11)$ \\
\hline $\mathrm{O}-\mathrm{Li}(1)-\mathrm{N}(2)$ & $140.9(11)$ & $N(2)-\operatorname{Li}(2)-N(1)$ & $166.3(12)$ \\
\hline$C(25)-O-\operatorname{Li}(1)$ & $125.2(10)$ & $\mathrm{C}(28)-\mathrm{O}-\mathrm{Li}(1)$ & $128.2(12)$ \\
\hline $\mathrm{C}(28)-\mathrm{O}-\mathrm{C}(25)$ & $105.8(12)$ & $C(2)-C(1)-\operatorname{Si}(1)$ & $112.5(9)$ \\
\hline$C(3)-C(1)-\operatorname{Si}(1)$ & $107.4(9)$ & $C(3)-C(1)-C(2)$ & $110.4(12)$ \\
\hline$C(4)-C(1)-S i(1)$ & $112.2(9)$ & $C(4)-C(1)-C(2)$ & $105.3(11)$ \\
\hline$C(4)-C(1)-C(3)$ & $109.1(1.1)$ & $C(6)-C(5)-S i(1)$ & $108.0(9)$ \\
\hline$C(7)-C(5)-\operatorname{Si}(1)$ & $114.8(10)$ & $C(7)-C(5)-C(6)$ & $107.8(11)$ \\
\hline$C(8)-C(5)-\operatorname{Si}(1)$ & $113.1(9)$ & $C(8)-C(5)-C(6)$ & $105.5(11)$ \\
\hline$C(8)-C(5)-C(7)$ & $107.2(11)$ & $\mathrm{C}(10)-\mathrm{C}(9)-\mathrm{N}(1)$ & $111.9(11)$ \\
\hline$C(11)-C(9)-N(1)$ & $109.7(11)$ & $C(11)-C(9)-C(10)$ & $107.4(11)$ \\
\hline$C(12)-C(9)-N(1)$ & $110.3(12)$ & $C(12)-C(9)-C(10)$ & $107.9(11)$ \\
\hline$C(12)-C(9)-C(11)$ & $109.5(12)$ & $C(14)-C(13)-\operatorname{Si}(2)$ & $108.3(9)$ \\
\hline$C(15)-C(13)-\operatorname{Si}(2)$ & $109.6(9)$ & $C(15)-C(13)-C(14)$ & $107.2(12)$ \\
\hline$C(16)-C(13)-\operatorname{Si}(2)$ & $112.1(10)$ & $C(16)-C(13)-C(14)$ & $107.0(11)$ \\
\hline$C(16)-C(13)-C(15)$ & $112.4(12)$ & $C(18)-C(17)-\operatorname{Si}(2)$ & $112.3(9)$ \\
\hline$C(19)-C(17)-S i(2)$ & $109.5(9)$ & $C(19)-C(17)-C(18)$ & $106.1(10)$ \\
\hline$C(20)-C(17)-S i(2)$ & $115.5(9)$ & $C(20) C(17) C(18)$ & $105.8(11)$ \\
\hline$C(20)-C(17)-C(19)$ & $107.1(11)$ & $C(22)-C(21)-N(2)$ & $107.5(10)$ \\
\hline$C(23)-C(21)-N(2)$ & $113.2(10)$ & $C(23)-C(21)-C(22)$ & $108.0(11)$ \\
\hline$C(24)-C(21)-N(2)$ & $112.4(11)$ & $C(24)-C(21)-C(22)$ & $106.2(11)$ \\
\hline$C(24)-C(21)-C(23)$ & $109.2(10)$ & $C(26)-C(25)-O$ & $107.1(18)$ \\
\hline$C(27)-C(26)-C(25)$ & $108.8(25)$ & $C(28)-C(27)-C(26)$ & $103.1(20)$ \\
\hline $\mathrm{C}(27)-\mathrm{C}(28)-\mathrm{O}$ & $111.9(18)$ & & \\
\hline
\end{tabular}

zeigt, dass das Signal bei 1.33 ppm vom NLiN-Lithiumatom und das Signal bei -0.18 ppm vom FLiF-Lithiumatom stammt. Die $J(\mathrm{LiF})$-Kopplung liegt bei $33 \mathrm{~Hz}$. Das Tieftemperatur- ${ }^{19} \mathrm{~F}-\mathrm{NMR}$-Spektrum zeigt auch bei $-72^{\circ} \mathrm{C}$ ein Singulett bei -0.3 ppm. Die Fluorkerne sind, anders als die Lithiumkerne, auch bei tiefer Temperatur magnetisch äquivalent. Vergleichbare Ergebnisse zeigen die NMRSpektren von 6. Ungewöhnlich ist bei $\mathbf{6}$ die chemische Verschiebung für den ${ }^{7} \mathrm{Li}-$ Kern mit $2.9 \mathrm{ppm}$, da ${ }^{7} \mathrm{Li}-\mathrm{NMR}-$ Verschichungen kovalent gebundener Lithiumorganyle normalerweise nur einen kleinen Bereich $( \pm 1 \mathrm{ppm})$ überstreichen. Die NMR-spektroskopischen Befunde zeigen, dass 5 und $\mathbf{6}$ in Lösung Moleküle mit 'fluktuierender' Bindung sind.

Da für Li(1) die Stickstoffatome N(1) und N(2) gleichwertig sind, kommt es in Iösung durch ein schnelles Öffnen der Li(1)-N(2)-Bindung und Bilden einer 
Tabelle 4

Wichtige kristallographische Daten der Verbindung 5

\begin{tabular}{|c|c|}
\hline Summenformel & $\mathrm{C}_{28} \mathrm{H}_{62} \mathrm{~F}_{2} \mathrm{Li}_{2} \mathrm{~N}_{2} \mathrm{OSi}_{2}$ \\
\hline$M$ & 550.86 \\
\hline Datensamml. bei $T\left({ }^{\circ} \mathrm{C}\right)$ & -80 \\
\hline Raumgruppe & $P 2_{1} / n$ \\
\hline$a(\mathrm{pm})$ & $1403.6(1)$ \\
\hline$b(\mathbf{p m})$ & $1224.3(4)$ \\
\hline$c(\mathrm{pm})$ & $2086.2(2)$ \\
\hline$\alpha\left({ }^{0}\right)$ & 90 \\
\hline$\beta\left({ }^{\circ}\right)$ & $95.25(12)$ \\
\hline$\gamma\left({ }^{\circ}\right)$ & 90 \\
\hline$V\left(\mathrm{~nm}^{3}\right)$ & 3.570 \\
\hline$Z$ & 4 \\
\hline$\rho_{\text {ber. }}\left(\mathrm{Mg} \mathrm{m}^{-3}\right)$ & 1.025 \\
\hline$\mu\left(\mathrm{mm}^{-1}\right)$ & 0.182 \\
\hline Kristallgrösse (mm) & $0.2 \times 0.2 \times 0.8$ \\
\hline \multicolumn{2}{|l|}{ Anzahl der Reflexe } \\
\hline gesammelte & 5860 \\
\hline unabhängige & 4648 \\
\hline beobachtete & 2322 \\
\hline$F>p \sigma(F)$ & $p=4$ \\
\hline $2 \Theta_{\max .}\left({ }^{\circ}\right)$ & 45 \\
\hline$R$ & 0.135 \\
\hline$R_{\mathrm{w}}$ & 0.119 \\
\hline$g$ & 0.0006 \\
\hline verfein. Parameter & 334 \\
\hline \multicolumn{2}{|l|}{ letzte Differenz-Fourier-Synthese } \\
\hline grösstes Maximum (e $\left.\mathrm{nm}^{-3}\right)$ & $6 \times 10^{3}$ \\
\hline grösstes Minimum (e nm ${ }^{-3}$ ) & $5.9 \times 10^{3}$ \\
\hline
\end{tabular}

Li(1)-N(1)-Bindung zu einer Äquilibrierung. Im Zeitmittel der NMR-Skala werden so die Fluor- und Kohlenstoffkerne magnetisch äquivalent, da das Molekül eine Spiegelebene besitzt. Die Lithiumkerne werden auch durch die Fluktuation der Li-N-Bindung magnetisch nicht äquivalent. Li(1) kann im Zeitmittel als fünffach koordiniert verstanden werden, $\operatorname{Li}(2)$ bleibt jedoch zweifach koordiniert. Die Struktur im Festkörper (Fig. 1 und 2) zeigt einen 'eingefrorenen' Zustand. Hier ist die Li-N-Bindung fixiert.

\section{Experimenteller Teil}

Die Versuche wurden unter trockenem $\mathrm{N}_{2}$ durchgeführt. NMR-Spektren (30 proz. Lösungen in n-Hexan, $C_{6} D_{6}$; TMS, $C_{6} F_{6}$ int.), Bruker AM-250 Kernresonanzgerät.

\section{Lithium-aminofluorsilane 5, 6}

0.01 mol 1 bzw. 2 in $40 \mathrm{ml} \mathrm{n-Hexan} \mathrm{werden} \mathrm{unter} \mathrm{N}_{2}$ mit $0.01 \mathrm{~mol} \mathrm{n}-\mathrm{C}_{4} \mathrm{H}_{9} \mathrm{Li}(15$ proz. in n-Hexan) lithiiert. Der Reaktionsfortschritt wird ${ }^{19}$ F-NMR-spektroskopisch verfolgt. Nach beendeter Reaktion werden die Lithiumsalze in THF und anschliessend $\mathrm{n}$-Hexan umkristallisiert. 


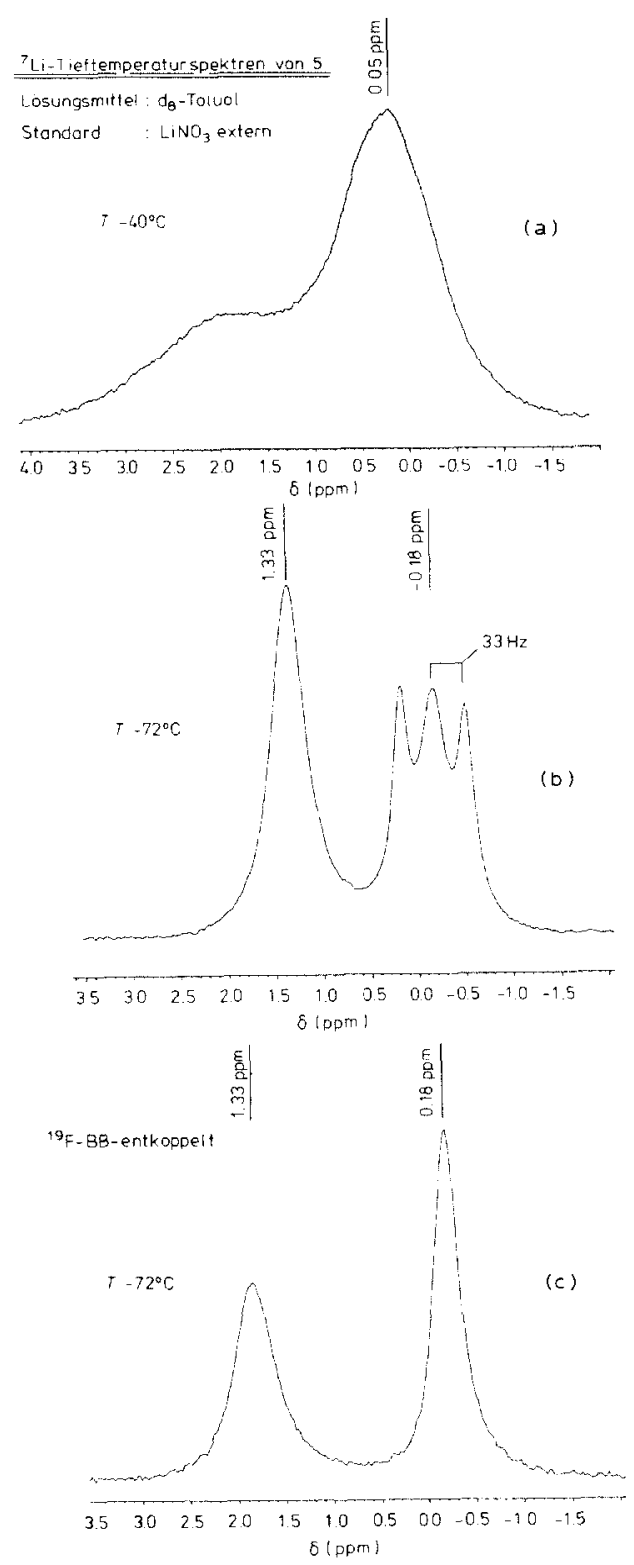

Fig. 3. ${ }^{7}$ Li-NMR-Tieftemperaturspektren von 5 (Lösungsmittel: Toluol-ds; Standard: LiNO 3 extern).

\section{Dank}

Diese Arbeit wurde von der Stiftung Volkswagenwerk und dem Fonds der Chemischen Industrie gefördert.

Herrn Finner von der Kali-Chemie Hannover danken wir für die Festkörper- ${ }^{13} \mathrm{C}$ NMR Messung von 5. 
Tabelle 5

${ }^{1} \mathrm{H}-,{ }^{13} \mathrm{C}-,{ }^{19} \mathrm{~F}-,{ }^{29} \mathrm{Si}-,{ }^{7} \mathrm{Li}-$ und ${ }^{14} \mathrm{~N}-\mathrm{NMR}-\mathrm{Daten}$ von 5 und 6

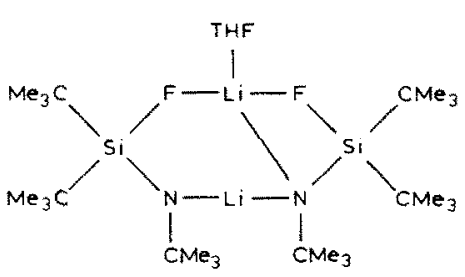

(5)

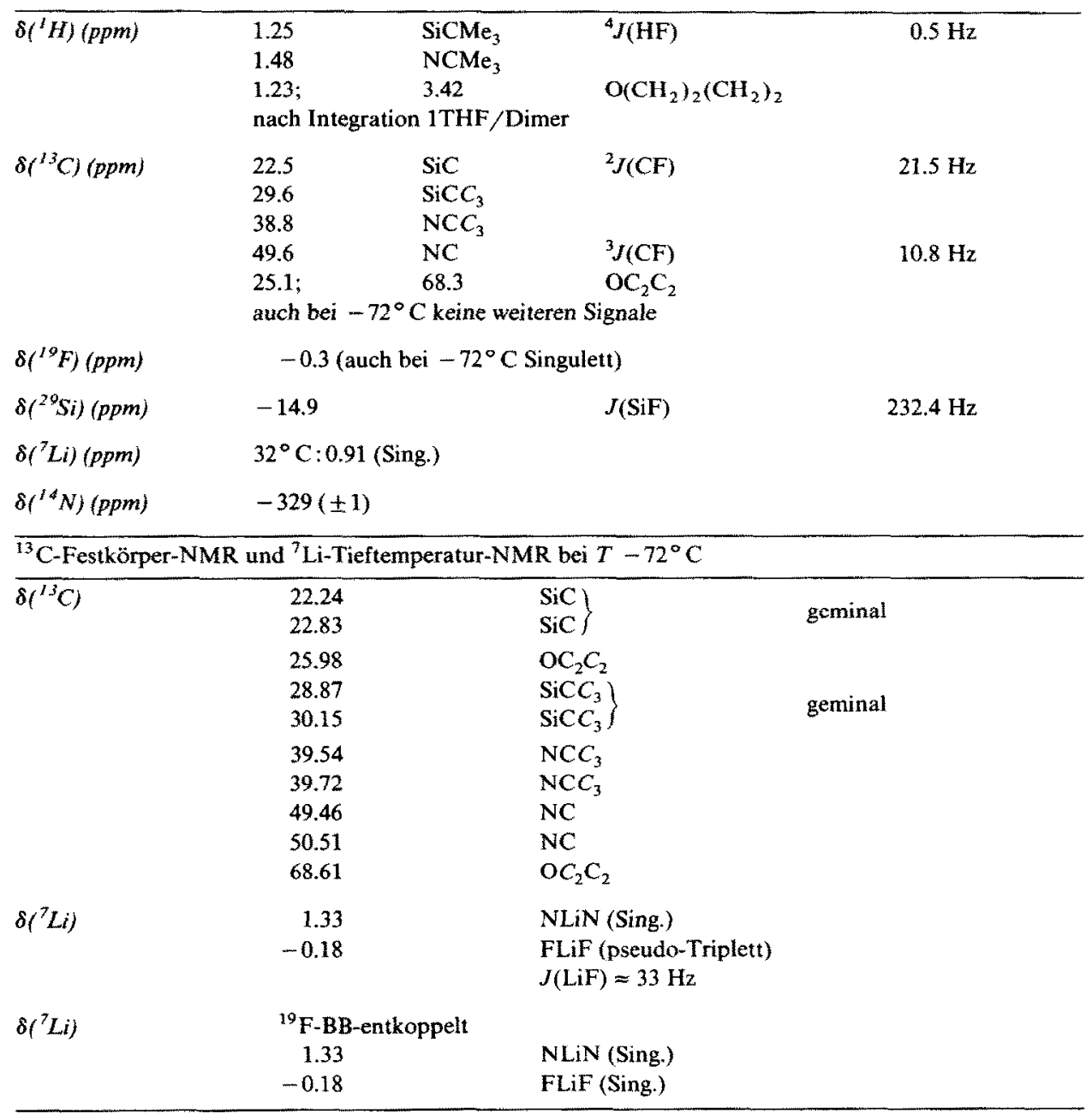




\begin{tabular}{|c|c|c|c|c|c|}
\hline$\left(\mathrm{Me}_{3} \mathrm{Si}^{\mathrm{Me}}\right)_{2}$ & $\operatorname{Me}_{3}^{\mathrm{C}}$ & ${ }^{H F}$ & $-(\overbrace{\operatorname{sime}_{3}}^{\mathrm{Ne}})$ & & \\
\hline$\overline{\delta\left({ }^{I} H\right)(p p m)}$ & $\begin{array}{l}0.24 \\
1.37 \\
2.62 \\
1.44 \\
\text { nach Int }\end{array}$ & $\begin{array}{l}\mathrm{SiMe}_{3} \\
\mathrm{CMe}_{3} \\
\mathrm{NMe} \\
3.56 \\
\text { ration } 1\end{array}$ & $\begin{array}{l}{ }^{5} J(\mathrm{HF}) \\
{ }^{4} J(\mathrm{HF}) 1.8 \mathrm{~Hz} \\
\mathrm{O}\left(\mathrm{CH}_{2}\right)_{2}\left(\mathrm{CH}_{2}\right)_{2} \\
\mathrm{HF} / \mathrm{Dimer}\end{array}$ & $1.1 \mathrm{~Hz}$ & $\begin{array}{l}\delta\left({ }^{7} \mathrm{Li}\right) 2.92 \text { ppm }\left(32^{\circ} \mathrm{C}\right) \\
\quad\left(\text { in } \mathrm{C}_{6} \mathrm{D}_{6}\right)\end{array}$ \\
\hline$\delta\left({ }^{1 / 3} C\right)(p p m)$ & $\begin{array}{l}0.52 \\
31.91 \\
37.31 \\
50.90 \\
25.37\end{array}$ & $\begin{array}{l}\mathrm{SiC} \\
\mathrm{NCC}_{3} \\
\mathrm{NCC}_{3} \\
\mathrm{NC} \\
68.53\end{array}$ & ${ }^{4} J(\mathrm{CF})$ & $1.8 \mathrm{~Hz}$ & $\begin{array}{l}\delta\left({ }^{7} \mathrm{Li}\right)\left(-80^{\circ} \mathrm{C}\right): \\
0.23 \mathrm{NLiN} \text { (Sing.) }\end{array}$ \\
\hline$\delta\left({ }^{l 9} F\right)(p p m)$ & 42.01 & & & & $\begin{array}{l}0.88 \text { FLiF (pseudo-Triplett) } \\
\text { (in Toluol- } d_{8} \text { ) }\end{array}$ \\
\hline$\delta\left({ }^{29} \mathrm{Si}\right)(\mathrm{ppm})$ & $\begin{array}{l}-45.01 \\
4.0\end{array}$ & $\begin{array}{l}\mathrm{SiF} \\
\mathrm{SiMe}_{3}\end{array}$ & $J(\mathrm{SiF})$ & $214.2 \mathrm{~Hz}$ & $J(\mathrm{LiF}) \approx 50 \mathrm{~Hz}$ \\
\hline
\end{tabular}

\section{Literatur}

1 M.F. Lappert, M.J. Slade, A. Singh, J.L. Atwood, R.D. Rogers und R. Shakir, J. Am. Chem. Soc., 105 (1983) 302 und dort zitierte Literatur.

2 D. Stalke, N. Keweloh, U. Klingebiel, M. Noltemeyer und G.M. Sheldrick, Z. Naturforsch., im Druck und dort zitierte Literatur.

3 D.J. Brauer, H. Bürger, G.R. Liewald und J. Wilke, J. Organomet. Chem., 287 (1985) 305.

4 D. Mootz, A. Zinnius und B. Böttcher, Angew. Chem. Int. Ed. Engl., 8 (1969) 378; R.D. Rogers, J.L. Atwood und R. Grüning, J. Organomet. Chem., 157 (1978) 229.

5 R. Boese und U. Klingebiel, J. Organomet. Chem., 315 (1986) C17.

6 U. Klingebiel und A. Meller, Angew. Chem. Int. Ed. Engl., 15 (1976) 313.

7 U. Klingebiel und J. Neemann, Z. Naturforsch. B, 35 (1980) 1155.

8 W.E. Rhine, G. Stucky und S.W. Peterson, J. Amer. Chem. Soc., 97 (1975) 6401. 